\title{
What our eyes tell us about feelings: Tracking pupillary responses during emotion regulation processes
}

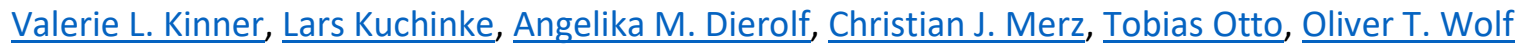

First published: 10 January 2017

\section{https://doi.org/10.1111/psyp.12816}

We gratefully acknowledge the help of Mira Beermann and Nuria Mackes during data collection and recruitment of participants. Funding for this study was provided by Project WO 733/15-1 of the German Research Foundation (DFG).

\begin{abstract}
Emotion regulation is essential for adaptive behavior and mental health. Strategies applied to alter emotions are known to differ in their impact on psychological and physiological aspects of the emotional response. However, emotion regulation outcome has primarily been assessed via self-report, and studies comparing regulation strategies with regard to their peripheral physiological mechanisms are limited in number. In the present study, we therefore aimed to investigate the effects of different emotion regulation strategies on pupil dilation, skin conductance responses, and subjective emotional responses. Thirty healthy females were presented with negative and neutral pictures and asked to maintain or up- and downregulate their upcoming emotional responses through reappraisal or distraction. Pupil dilation and skin conductance responses were significantly enhanced when viewing negative relative to neutral pictures. For the pupil, this emotional arousal effect manifested specifically late during the pupillary response. In accordance with subjective ratings, increasing negative emotions through reappraisal led to the most prominent pupil size enlargements, whereas no consistent effect for downregulation was found. In contrast, early peak dilations were enhanced in all emotion regulation conditions independent of strategy. Skin conductance responses were not further modulated by emotion regulation. These results indicate that pupil diameter is modulated by emotional arousal, but is initially related to the extent of mental effort required to regulate automatic emotional responses. Our data thus provide first evidence that the pupillary response might comprise two distinct temporal components reflecting cognitive emotion regulation effort on the one hand and emotion regulation success on the other hand.
\end{abstract}

